\title{
Einfluss der Rotorgeometrie auf Schwingungs- und Geräuschemissionen elektrischer Fahrzeugantriebe
}

\author{
M. EnglandD, B. Ponick
}

\begin{abstract}
Permanentmagneterregte Synchronmaschinen werden im Bereich der Traktionsmaschinen in der Regel mit einer Rotorstaffelung ausgeführt, d. h. der Rotor wird ähnlich wie bei der Nutschrägung entlang des Blechpakets segmentweise um insgesamt eine Nutteilung am Umfang versetzt, um Parasitäreffekte wie Drehmomentwelligkeit zu reduzieren und das akustische Verhalten zu verbessern. Allerdings wird durch die Staffelung der Rotorsegmente in axialer Richtung die Hauptwellenflussverkettung der Permanentmagnete gegenüber einer ungestaffelten Maschine verringert. Daher muss in einer gestaffelten Maschine mehr Magnetmaterial eingesetzt werden als in einer ungestaffelten Maschine, um das gleiche Drehmoment zu erreichen, was sich in den Kosten niederschlägt. Darüber hinaus ist die Staffelung ein vergleichsweise aufwendiger Schritt im Fertigungsprozess, sodass sich die Produktionszeit je Maschine erhöht und letztendlich die Kosten steigen.

In diesem Artikel wird der Einfluss einer Rotorpolweitenmodulation auf das Betriebsverhalten für V-förmig vergrabene Magnettopologien untersucht. Es wird bewertet, ob mithilfe dieser zusätzlichen Modifikation der Maschinengeometrie ein Antrieb ohne Staffelung ausgeführt werden kann, der die Vorgaben des Lastenhefts in Bezug auf die Drehmomentwelligkeit sowie auf das maximale Drehmoment einhält und gleichzeitig die Geräuschemissionen begrenzt. Zur vorteilhaften Gestaltung einer Rotorpolweitenmodulation wird eine Kombination der Magnetöffnungswinkel mittels eines analytischen Netzwerks identifiziert, die die für die Anregung von Pendelmomenten und Geräuschen kritischen Feldwellen des Rotors reduzieren kann. Anschließend werden die analytischen Ergebnisse mit FEM-Berechnungen validiert. Der Einfluss der Rotorpolweitenmodulation auf das Drehmomentverhalten sowie die Geräuschanregung wird mit der gestaffelten Referenzmaschine verglichen.
\end{abstract}

Schlüsselwörter: Traktionsantrieb; Permanentmagneterregte Synchronmaschine (PMSM); Geräuschemission; Drehmomentwelligkeit; asymmetrischer Rotor

Influence of rotor geometry on vibrations and acoustic noise emissions of electric traction machines.

In the field of traction machines, permanent-magnet synchronous machines are usually designed with step-skewed rotors, i. e., the rotor is skewed along the core length segment by segment by one slot pitch in order to reduce parasitic effects, such as torque ripple, and to improve the acoustic behavior. However, step-skewing the rotor segments in the axial direction reduces the fundamental flux linkage of the permanent magnets compared to an unskewed machine. Therefore, more magnet material must be used in a skewed machine than in an unskewed machine to achieve the same torque, which increases the cost. Furthermore, skewing is a comparatively complex step in the manufacturing process, which increases the production time per machine and the cost.

In this article, the influence of rotor pole width modulation on the performance of $v$-shaped buried magnet topologies is investigated. It evaluates whether this additional modification to the machine geometry can be used to create an unskewed motor that meets the specifications for torque ripple and maximum torque while limiting noise emissions. In order to design the rotor pole width modulation in a profitable way, a combination of magnet angles is identified by an analytical network that can reduce the critical flux density spatial harmonics of the rotor, which stimulate torque ripple and acoustic noise. Afterwards, the analytical results are validated with FEM calculations. The effect of the rotor pole width modulation on torque behavior as well as acoustic noise emission is compared with the step-skewed reference machine.

Keywords: traction; permanent-magnet synchronous machine (PMSM); NVH; acoustic noise emission; torque ripple; asymmetric rotor

Eingegangen am 18. Mai 2021, angenommen am 25. Juni 2021, online publiziert am 13. Juli 2021

(c) The Author(s) 2021

\section{Formelzeichen}

$B_{\text {rad }} \quad$ radiale Grenzflächeninduktion

$B_{\tan } \quad$ tangentiale Grenzflächeninduktion

b $\quad$ Magnetbreite

$b_{\sigma} \quad$ Streustegbreite

$d_{\mathrm{a} 1} \quad$ Statoraußendurchmesser

$d_{\mathrm{i} 1} \quad$ Statorinnendurchmesser

$f \quad$ Frequenz

$f_{\text {eigen } \quad \text { Eigenfrequenz }}$

$h_{\mathrm{PM}} \quad$ Magnethöhe

$\begin{array}{ll}h_{\text {spalt }} & \text { Höhe des Fügespalts } \\ l & \text { Stangstrom } \\ L_{p, \text { ges }} & \text { Gesamtschalldruckpegel } \\ l_{\text {ax }} & \text { Blechpaketlänge }\end{array}$

England, Marc, Institut für Antriebssysteme und Leistungselektronik, Leibniz Universität Hannover, Welfengarten 1, 30167 Hannover, Deutschland

(E-Mail: marc.england@ial.uni-hannover.de). Ponick, Bernd, Institut für Antriebssysteme und Leistungselektronik, Leibniz Universität Hannover, Hannover, Deutschland 


$\begin{array}{ll}M & \text { Drehmoment } \\ m & \text { Strangzahl } \\ N & \text { Nutzahl } \\ n & \text { Drehzahl } \\ p & \text { Polpaarzahl } \\ q & \text { axiale Schwingungsmode } \\ R & \text { magnetischer Widerstand } \\ r & \text { radiale Schwingungsmode } \\ r_{\delta, \text { mid }} & \text { mittlerer Luftspaltradius } \\ t & \text { Zeit } \\ w & \text { Strangwindungszahl } \\ \beta & \text { Anstellwinkel } \\ \gamma^{\prime} & \text { Winkelumfangskoordinate } \\ \Delta B & \text { Versatzfaktor der Induktion } \\ \Delta M & \text { relative Drehmomentwelligkeit } \\ \delta & \text { Luftspalthöhe } \\ \mu_{0} & \text { magnetische Feldkonstante } \\ \mu_{r} & \text { relative Permeabilität } \\ \mu^{\prime} & \text { Ordnungszahl des Strombelags } \\ v^{\prime} & \text { Ordnungszahl des Induktion } \\ v_{\mathrm{NH}}^{\prime} & \text { Ordnungszahl der Nutungsharmonischen } \\ \xi & \text { Wicklungsfaktor } \\ \sigma_{\text {rad }} & \text { radiale Zugspannungswelle } \\ \sigma_{\text {tan }} & \text { tangentiale Zugspannungswelle } \\ \varphi & \text { Phasenversatz } \\ \Phi & \text { magnetischer Fluss } \\ \chi & \text { Oberwellengehalt } \\ \omega & \text { Kreisfrequenz } \\ & \end{array}$

\section{Einleitung}

Die Traktionsantriebe in der Automobilindustrie werden oftmals als permanentmagneterrgete Synchronmaschinen (PMSM) ausgeführt, da diese den Vorteil einer hohen Drehmomentdichte sowie eines hohen Wirkungsgrads innerhalb eines geringen Bauraums aufweisen. Bei der Dimensionierung dieser Antriebe sind darüber hinaus Parasitäreffekte wie Pendelmomente, Vibrationen und daraus resultierende Geräuschemissionen zu berücksichtigen. Die Ursache für diese Parasitäreffekte sind periodische Tangential- und Radialkräfte, die auf den Stator und den Rotor wirken [1]. Die Tangentialkräfte erzeugen das konstante Drehmoment sowie die Pendelmomente der Maschine, während die an den Statorzähnen angreifenden Radialkräfte zur Verformung des Stators führen. Die dadurch erregten Vibrationen des Statorblechpakets resultieren in unerwünschten Geräuschemissionen.

Da das resultierende Drehmoment aus einer Wegintegration der Tangentialspannungen über die gesamte Bohrungsfläche resultiert, wird in der Regel eine kontinuierliche Schrägung oder eine segmentweise Staffelung des Rotors bzw. des Stators ausgeführt, um die Pendelmomente zu reduzieren [2, 3]. Eine Schrägung des Stators führt zu einem erhöhten Aufwand bei der Fertigung der Wicklung. Vor allem bei im Automotive-Bereich zunehmenden Einsatz von Formleitern wie z.B. Haarnadelwicklungen ist diese Variante unerwünscht [4]. Daher wird üblicherweise der Rotor einer PMSM gestaffelt ausgeführt, wobei die Anordnung der Staffelungssegmente einen Einfluss auf das Vibrationsverhalten aufweist. So kann gezeigt werden, dass eine V-Staffelung oder eine Zickzack-Staffelung Vorteile gegenüber einer linearen Staffelung besitzen [5, 6]. Allerdings führt die Ausführung einer Staffelung zur einer Reduktion der Hauptwellenflussverkettung des Rotorfelds mit der Statorwicklung. Somit muss mehr Magnetmaterial eingesetzt werden, um das gleiche Drehmoment wie bei einer ungestaffelten Maschine zu erreichen. Außerdem erhöht sich die Produktionszeit je Maschine im
Fertigungsprozess. Beides führt schließlich zu einer Steigerung der Kosten.

Es existieren unterschiedliche Ansätze zur Reduktion von Pendelmomenten und Schwingungsanregungen. So können ungleiche Zahnbreiten in Maschinen mit Zahnspulenwicklung zur Reduktion der Pendelmomente genutzt werden [7]. Zusätzliche Einkerbungen oder Ausprägungen an den Statorzähnen führen zur Reduktion von Rastmomenten [8]. In PMSM mit vergrabener V-förmiger Magnettopologie wirkt sich ein asymmertrisches $V$ mit unterschiedlichen Magnetlängen innerhalb eines Pols positiv auf die Drehmomentwelligkeit aus [9]. Außerdem kann die Form der Flussperren am Rotoraußendurchmesser angepasst werden [10], oder diese können asymmetrisch aufgebaut werden [11, 12]. Darüber hinaus kann die Anpassung der Oberflächenkontur des Rotors zur Reduktion der Oberfeldanteile im Luftspaltfeld genutzt werden [13-15]. Eine weitere Möglichkeit ist die Ausführung asymmetrischer Polpaare mithilfe einer Rotorpolweitenmodulation (RPWM). Dies ist bereits für eine Permanentmagnet unterstützte Reluktanzmaschine [16] sowie für eine fünfphasige PMSM mit V-Magneten untersucht worden [17]. Ein alternativer Ansatz über die Leistungselektronik ist die Einspeisung zusätzlicher Oberschwingungsströme mithilfe eines Frequenzumrichters zur Reduktion der Anregung der Atemmode [18]

Da ein Großteil der Veröffentlichungen sich ausschließlich mit der Reduktion von Pendelmomenten im Leerlauf und in bestimmten Arbeitspunkten beschäftigt, wird in diesem Artikel der Einfluss einer Rotorpolweitenmodulation im gesamten Betriebsbereich einer PMSM untersucht und zusätzlich die Auswirkung der Modifikationsvariante auf die Geräuschanregungen betrachtet. Dazu werden zunächst die Ursprünge der magnetischen Kräfte dargestellt, bevor ein vereinfachtes analytisches Ersatznetzwerk zur Bestimmung des Induktionsverlaufs im Luftspalt aufgrund des Rotorfelds vorgestellt wird. Die Modellierung orientiert sich an Vorarbeiten von [17, 19] und erlaubt die Identifikation vorteilhafter Winkelkombinationen für eine Rotorpolweitenmodulation. Anschließend erfolgt der Vergleich einer modifizierten Maschine mit der Referenzmaschine mithilfe von FEM-Simulationen. So wird der Einfluss auf das Betriebsverhalten im gesamten Betriebsbereich analysiert. Abschließend wird eine Berechnungsmethodik zur Bewertung des akustischen Verhaltens der Maschinen vorgestellt und ein Vergleich zwischen der Referenzmaschine und einer Maschine mit RPWM wird dargestellt.

\section{Magnetische Kräfte und Drehmomentbildung}

Auf Grundlage von Maxwell'schen Grenzflächenkräften kann mittels des Spannungstensors die Zugspannung in radialer Richtung zu

$$
\sigma_{\mathrm{rad}}=\frac{B_{\mathrm{rad}}^{2}-B_{\mathrm{tan}}^{2}}{2 \mu_{0}}
$$

und in tangentialer Richtung zu

$$
\sigma_{\mathrm{tan}}=\frac{B_{\mathrm{rad}} \cdot B_{\mathrm{tan}}}{\mu_{0}}
$$

bestimmt werden [20, 21]. Darin beschreibt $B_{\text {rad }}$ die Grenz-flächeninduktion in radialer Richtung und $B_{\tan }$ die Grenzflächeninduktion in tangentialer Richtung an einer Grenzfläche zwischen Elektroblech und Luftspalt sowie $\mu_{0}$ die magnetische Feldkonstante. Wird das resultierende Luftspaltfeld als Summe von Drehwellen

$$
B\left(\gamma^{\prime}, t\right)=\sum_{v^{\prime}} \hat{B}_{v^{\prime}} \cos \left(v^{\prime} \gamma^{\prime}-2 \pi f_{v^{\prime}} t+\varphi_{v^{\prime}}\right)
$$

beschrieben, worin $\gamma^{\prime}$ die Winkelumfangskoordinate, $t$ die Zeit, $v^{\prime}$ die Ordnungszahl, $f_{v}^{\prime}$ die Frequenz und $\varphi_{v^{\prime}}$ den Phasenversatz bezeichnen, können die entstehenden Zugspannungswellen ermittelt 

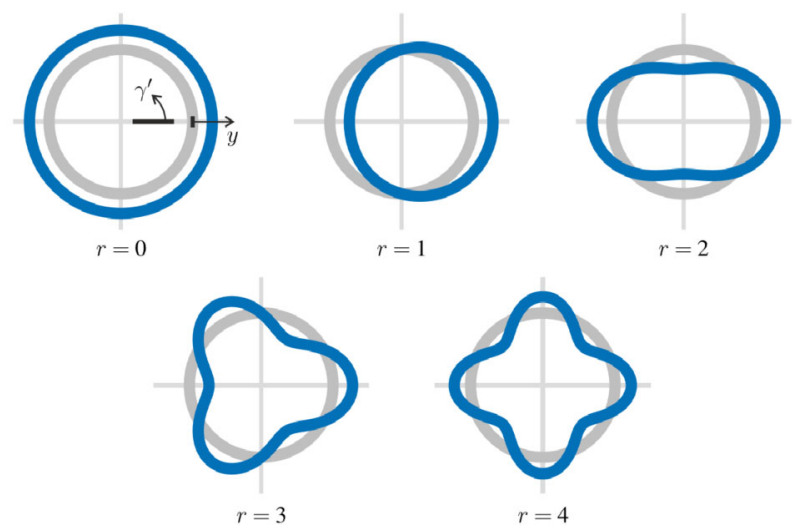

Abb. 1. Die ersten fünf Schwingungsmoden eines Kreisrings [23]

werden. Bei einem radialen Feldverlauf mit $B_{\tan }=0$ ergeben sich aus zwei Drehwellen mit den Ordnungszahlen $v_{1}^{\prime}$ und $v_{2}^{\prime}$ mit den Kreisfrequenzen $\omega_{v^{\prime}}=2 \pi f_{v^{\prime}}$ die radialen Zugspannungswellen

$$
\begin{aligned}
\sigma_{\text {rad }}\left(\gamma^{\prime}, t\right) & = \\
& =\frac{\hat{B}_{v^{\prime} 1}^{2}}{4 \mu_{0}} \cdot\left(1+\cos \left(2 v_{1}^{\prime} \gamma^{\prime}-\omega_{v^{\prime} 1}+2 \varphi_{v^{\prime} 1}\right)\right) \\
& +\frac{\hat{B}_{v^{\prime}}^{2}}{4 \mu_{0}} \cdot\left(1+\cos \left(2 v_{2}^{\prime} \gamma^{\prime}-\omega_{v^{\prime} 2}+2 \varphi_{v^{\prime} 2}\right)\right) \\
& +\frac{\hat{B}_{v^{\prime} 1} \hat{B}_{v^{\prime} 2}}{2 \mu_{0}} \cdot \cos \left[\left(v_{2}^{\prime}+v_{1}^{\prime}\right) \gamma^{\prime}\right. \\
& \left.-\left(\omega_{v^{\prime} 2}+\omega_{v^{\prime} 1}\right)+\left(\varphi_{v^{\prime} 2}+\varphi_{v^{\prime} 1}\right)\right] \\
& +\frac{\hat{B}_{v^{\prime} 1} \hat{B}_{v^{\prime} 2}}{2 \mu_{0}} \cdot \cos \left[\left(v_{2}^{\prime}-v_{1}^{\prime}\right) \gamma^{\prime}\right. \\
& \left.-\left(\omega_{v^{\prime} 2}-\omega_{v^{\prime} 1}\right)+\left(\varphi_{v^{\prime} 2}-\varphi_{v^{\prime} 1}\right)\right] .
\end{aligned}
$$

Für die Geräuschemission sind die periodischen Kraftanregungen maßgeblich, deren modale Ordnungen und Frequenzen aus der Addition bzw. der Differenz der Argumente zweier beteiligter Feldwellen entstehen [21]. Für diese wechselwirkenden Induktionswellen ergeben sich Zugspannungswellen mit der räumlichen modalen Ordnung

$$
r=v_{2}^{\prime} \pm v_{1}^{\prime}
$$

und der Anregungsfrequenz

$$
f_{r}=f_{v 2^{\prime}} \pm f_{v 1^{\prime}}
$$

Hieraus ist zu erkennen, dass auch die Wechselwirkung zweier hochpoliger Induktionswellen zu Kraftanregungen mit niedriger räumlicher Ordnung führen kann. Typischerweise ist die Anregung der sogenannten Atemmode mit der räumlichen Ordnung $r=0$ die dominante Quelle für Geräuschemissionen bei Fahrzeugantrieben [22]. Mit Zunahme der räumlichen Ordnung steigt die Steifigkeit des Blechpakets. Die Anregung von Moden höherer räumlicher Ordnung wird daher erschwert [23]. Räumliche Verformungsordnungen für ein Statorjoch als Kreisring sind exemplarisch in Abb. 1 dargestellt.

Zur Bildung des Drehmoments erfolgt eine Integration der tangentialen Zugspannungswellen über die axiale Länge $l_{a x}$ sowie über den Umfang und eine Multiplikation mit dem Bohrungsradius $R$.
Dies kann ebenso durch eine Multiplikation des Statorstrombelags $A\left(\gamma^{\prime}, t\right)$ mit der Luftspaltinduktion

$$
\begin{aligned}
M(t) & =R l_{\mathrm{ax}} \int_{0}^{2 \pi} \sigma_{\tan }\left(\gamma^{\prime}, t\right) R \mathrm{~d} \gamma^{\prime} \\
& =R^{2} l_{\mathrm{ax}} \int_{0}^{2 \pi} A\left(\gamma^{\prime}, t\right) B\left(\gamma^{\prime}, t\right) \mathrm{d} \gamma^{\prime}
\end{aligned}
$$

beschrieben werden [20]. Dafür liegt der Strombelag als Summe von Drehwellen

$$
A\left(\gamma^{\prime}, t\right)=\sum_{\mu^{\prime}} \hat{A}_{\mu^{\prime}} \cos \left(\mu^{\prime} \gamma^{\prime}-2 \pi f_{\mu^{\prime}} t+\varphi_{\mu^{\prime}}\right)
$$

vor, sodass analog zu Gleichung (4) eine Summe aus Cosiunstermen entsteht, welche dann integriert wird. Für die Wechselwirkung einer einzelnen Feldwelle des Strombelags mit der Ordnung $\mu^{\prime}$ mit einer einzelnen Drehwelle der Induktion $v^{\prime}$ können die folgenden Aussagen getroffen werden:

- Für $\mu^{\prime} \pm v^{\prime} \neq 0$ ergibt sich für das Integral der Wert 0. Die Wechselwirkung unterschiedlicher Ordnungszahlen liefert demnach keinen Beitrag zum resultierenden Drehmoment, jedoch entstehen lokale Tangentialspannungen.

- Für $\mu^{\prime} \pm v^{\prime}=0$ und $f_{\mu^{\prime}} \pm f_{v^{\prime}} \neq 0$ entsteht ein reines zeitlich veränderliches Pendelmoment der Frequenz $f_{\mu^{\prime}} \pm f_{v^{\prime}}$, das keinen Beitrag zum mittleren Drehmoment liefert.

- Für $\mu^{\prime} \pm v^{\prime}=0$ und $f_{\mu^{\prime}} \pm f_{v^{\prime}}=0$ haben die beiden Drehwellen dieselbe Ordnungszahl und keine Relativgeschwindigkeit zueinander, sodass ein konstantes Drehmoment entsteht.

Typischerweise entstehen hohe Pendelmomente in Maschinen mit Ganzlochwicklungen aufgrund der Interaktion sogenannter Nutund Nutungsharmonischer des Luftspaltfelds mit der Ordnungszahl

$$
v_{\mathrm{NH}}^{\prime}=g N \pm p \operatorname{mit} g \in \mathbb{N}
$$

mit den entsprechenden Strombelagswellen [21]. Somit ist es sinnvoll, durch eine Modifikation der Rotorgeometrie die Feldanteile mit dieser Ordnungzahl des Rotorfelds zu reduzieren, um die resultierende Drehmomentwelligkeit zu verringern.

\section{Magnetisches Ersatznetzwerk zur Bestimmung des Rotorfelds}

Die Ausführung eines Rotors mit variierenden Anstellwinkeln der Vförmig vergrabenen Magneten von Pol zu Pol ermöglicht die Beeinflussung des Luftspaltfelds, das vom Rotor erzeugt wird. Somit können die Feldanteile verringert werden, die zu Pendelmomenten und Geräuschanregungen führen. Diese Modifikation ist in Abb. 2 dargestellt und wird im weiteren Rotorpolweitenmodulation genannt. Zur Identifikation vorteilhafter Winkelkombinationen wird zunächst ein vereinfachtes analytisches Ersatznetzwerk erstellt. Dieses wird mit FEM-Berechnungen für eine Maschine mit symmetrischen Polen sowie für eine Maschine mit einer Rotorpolweitenmodulation validiert. Anschließend werden Gütekriterien vorgestellt und anhand dieser eine vorteilhafte Winkelkombination zur genaueren Untersuchung ausgewählt.

\subsection{Aufbau des magnetischen Ersatznetzwerks}

Zur zeiteffizienten Bewertung des Einflusses einer Variation der Anstellwinkel auf das Rotorfeld im Leerlauf wird ein magnetisches Ersatznetzwerk nach den Vorarbeiten aus [17, 19] erstellt. Da die Beeinflussung des Rotorfelds durch eine RPWM im Vordergrund steht, wird die Statornutung sowie die Sättigung im Stator zunächst nicht 


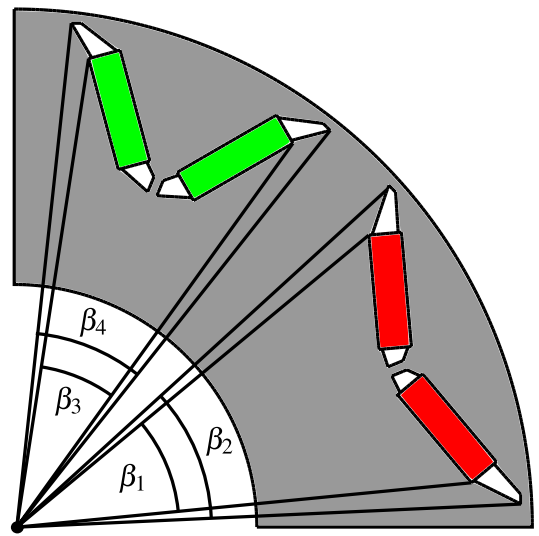

Abb. 2. Prinzipskizze einer Rotorpolweitenmodulation mit den Winkeldefinitionen

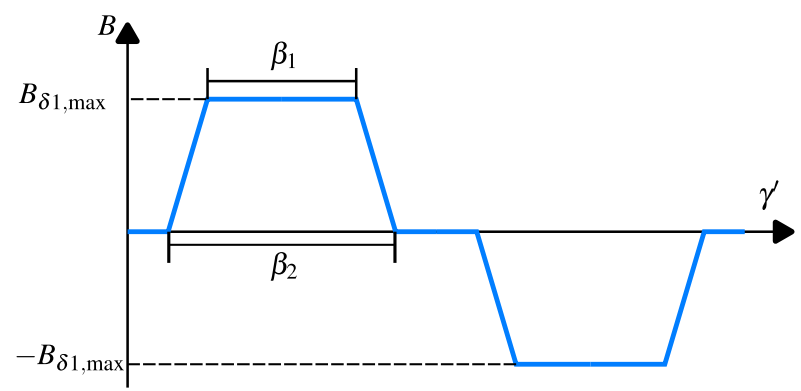

Abb. 3. Induktionsverlauf des Luftspaltfelds mit symmetrischen Polen

berücksichtigt. Wie in Abb. 2 dargestellt, beschreiben $\beta_{1}$ und $\beta_{3}$ den eingeschlossenen Winkel der Magnete und $\beta_{2}$ und $\beta_{4}$ den eingeschlossenen Winkel der luftspaltnahen Flusssperren des jeweiligen Pols. Für eine Maschine mit symmetrischer Anordnung gilt $\beta_{1}=\beta_{3}$ sowie $\beta_{2}=\beta_{4}$. Ein rein radial gerichtetes Luftspaltfeld des Rotors kann für $\mathrm{V}$-förmig vergrabene Magnettopologien mithilfe eines trapezförmigen Verlaufs beschrieben werden, wie er in Abb. 3 dargestellt ist [19]. Der Induktionsverlauf lässt sich in drei Bereiche aufteilen. Die Pollücke ist feldfrei. Im Bereich der gesättigten Flusssperren wird ein linearer Verlauf der Induktionsamplitude angenommen. Das Maximum bzw. Minimum der Induktion mit $B_{\delta, \max }$ ist innerhalb des Polbedeckungsgrads $\beta_{1}$ konstant. Da die Bestimmung der Anstellwinkel aus den geometrischen Maschinendaten erfolgen kann, wird ein magnetisches Ersatznetzwerk zur Bestimmung der Induktion $B_{\delta, \max }$ erstellt.

Anhand der Feldlinien in Abb. 4, die aus einer magnetostatischen Leerlaufrechnung identifiziert worden sind, kann der Fluss der Permanentmagnete in drei Anteile aufgespalten werden. Dies ist zum einen der Luftspaltfluss, der sich über den Luftspalt sowie den Stator ausbildet. Zum anderen können die Streuflüsse über die Streustege zwischen den Magneten mit der Breite $b_{\sigma 2}$ sowie über die luftspaltnahen Streustege mit der Breite $b_{\sigma 1}$ identifiziert werden. Unter der Annahme, dass im Leerlauf nur die Streustege gesättigt sind, werden die magnetischen Widerstände für Rotorjoch und Statorjoch vernachlässigt. Daraus resultiert das magnetische Ersatznetzwerk in Abb. 5. Darin wird der Permanentmagnet als ideale Flussquelle $\Phi_{P M}$ mit Parallelwiderstand $R_{\mathrm{PM}}$ dargestellt, und es werden Ersatzwiderstände für die Streustege $R_{\sigma 1}$ sowie $R_{\sigma 2}$ unter der Annahme, dass in diesen eine konstante Flussdichte von $B_{\text {sat }}=2,1 \mathrm{~T}$ vorherrscht,

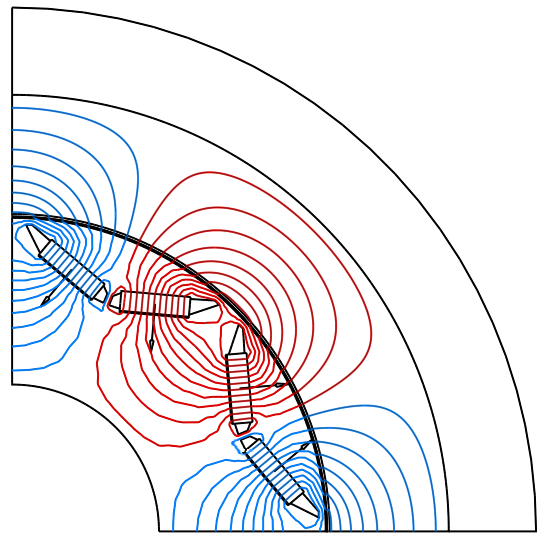

Abb. 4. Feldlinien einer Leerlaufrechnung mit ungenutetem Stator als Rückschluss

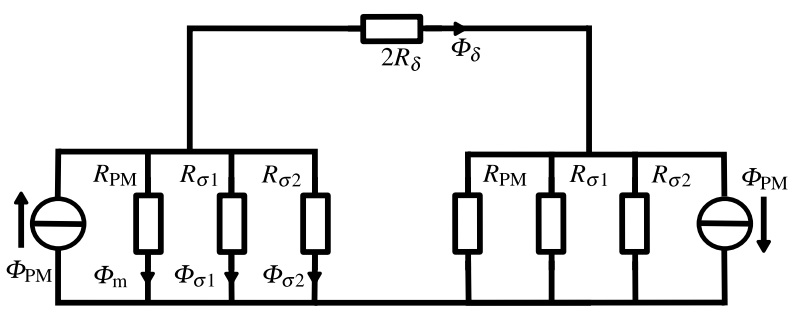

Abb. 5. Magnetisches Ersatznetzwerk einer Polteilung

ermittelt. Zusätzlich wird der Widerstand $R_{\mathrm{PM}, \text { Luft }}$ unterhalb der Magnete, der den Fügespalt zum Verkleben der Magnete im Rotorblech berücksichtigt, hinzugefügt. Der Luftspaltfluss $\Phi_{\delta}$ schließt sich über den Widerstand des Luftspalts $R_{\delta}$. Mithilfe des magnetischen Ersatznetzwerks können die Knoten- und Maschensätze

$$
\begin{aligned}
& \Phi_{\mathrm{PM} 1}=\Phi_{\mathrm{m} 1}+\Phi_{\sigma 1}+\Phi_{\sigma 2}+\Phi_{\delta 1} \\
& R_{\sigma 1} \Phi_{\sigma 1}-R_{\mathrm{PM} 1} \Phi_{\mathrm{m} 1}=0 \\
& 2 R_{\delta} \Phi_{\delta}-2 R_{\sigma 1} \Phi_{\sigma 1}=0
\end{aligned}
$$

aufgestellt werden. Die Streuflüsse werden über

$$
\begin{aligned}
& \Phi_{\sigma 1}=B_{\text {sat }} A_{\sigma 1}=B_{\text {sat }} b_{\sigma 1} l_{\text {ax }} \\
& \Phi_{\sigma 2}=B_{\text {sat }} A_{\sigma 2}=B_{\text {sat }} \frac{b_{\sigma 2}}{2} l_{\mathrm{ax}}
\end{aligned}
$$

abgeschätzt und die magnetischen Widerstände über

$$
\begin{aligned}
& R_{\mathrm{PM} 1}=\frac{h_{\mathrm{PM} 1}}{\mu_{0} \mu_{r} b_{\mathrm{PM} 1} l_{\mathrm{ax}}}+\frac{h_{\mathrm{Spalt}}}{\mu_{0} b_{\mathrm{PM} 1} l_{\mathrm{ax}}} \\
& R_{\delta 1}=\frac{\delta}{\mu_{0} A_{\delta 1}} \\
& \text { mit } A_{\delta 1}=\frac{1}{2} \frac{\beta_{1}+\beta_{2}}{2} r_{\delta, \text { mid }} l_{\mathrm{ax}}
\end{aligned}
$$

für einen Polbedeckungsgrad mit $\beta_{1}$ sowie für eine Magnethöhe $h_{\mathrm{PM} 1}$ und für eine Magnetbreite $b_{\mathrm{PM} 1}$ bestimmt, worin $\mu_{0}$ die magnetische Feldkonstante und $\mu_{\mathrm{r}}$ die relative Permeabilität des Magneten darstellen [17]. $h_{\text {spalt }}$ beschreibt die Höhe des Fügespalts unterhalb des Magneten und $r_{\delta \text {,mid }}$ den mittleren Luftspaltradius. Der Magnetfluss der Quelle wird mit der Remanzflussdichte im Leerlauf 


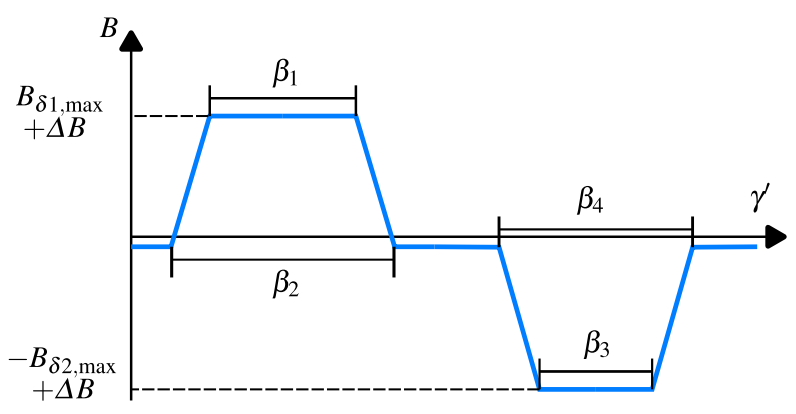

Abb. 6. Induktionsverlauf des Luftspaltfelds mit asymmetrischen Polen

über

$$
\Phi_{\mathrm{PM} 1}=\frac{B_{\mathrm{R}} h_{\mathrm{PM} 1}}{\mu_{0} \mu_{r} R_{\mathrm{PM} 1}}
$$

bestimmt. So ergibt sich schließlich die maximale Flussdichte im Luftspalt zu

$$
B_{\delta 1, \max }=\frac{\Phi_{\delta 1}}{A_{\delta 1}}
$$

mit

$$
\Phi_{\delta 1}=\frac{\Phi_{\mathrm{PM} 1}-\Phi_{\sigma 1}-\Phi_{\sigma 2}}{1+\frac{R_{\delta 1}}{R_{\mathrm{PM} 1}}}
$$

\subsection{Aufbau des magnetischen Ersatznetzwerks für}

Rotorpolweitenmodulation

Der grundsätzliche Verlauf der Feldlinien wird aufgrund einer Rotorpolweitenmodulation über die Anstellwinkel der Magnete nur geringfügig beeinflusst. Daher ist es zulässig, das magnetische Ersatznetzwerk auch auf eine Maschine mit RPWM anzuwenden [17]. Aufgrund der unterschiedlichen Polbedeckungsgrade in Pol 1 und Pol 2 mit $\beta_{1}$ bzw. $\beta_{3}$ ist jedoch eine Anpassung der maximalen Luftspaltinduktion notwendig. Zusätzlich verschiebt sich die Verlauf der Luftspaltinduktion um $\Delta B$, damit eine Integration der Flussdichte über eine doppelte Polteilung null ergibt. So resultiert der Verlauf einer Luftspaltinduktion mit RPWM, wie er in Abb. 6 dargestellt ist. Für den ersten Pol können die Gleichungen aus dem Abschn. 3.1 genutzt werden, während für den zweiten Pol die magnetischen Widerstände

$$
\begin{aligned}
& R_{\mathrm{PM} 2}=\frac{h_{\mathrm{PM} 2}}{\mu_{0} \mu_{r} b_{\mathrm{PM} 2} l_{\mathrm{ax}}}+\frac{h_{\mathrm{Spalt}}}{\mu_{0} b_{\mathrm{PM} 2} l_{\mathrm{ax}}} \\
& R_{\delta 2}=\frac{\delta}{\mu_{0} A_{\delta 2}} \\
& \text { mit } A_{\delta 2}=\frac{1}{2} \frac{\beta_{3}+\beta_{4}}{2} r_{\delta, \text { mid }} l_{\mathrm{ax}}
\end{aligned}
$$

berechnet werden. Schließlich wird $\Phi_{\mathrm{PM} 2}$ analog zu (15) bestimmt und bei gleicher Ausführung der Streustege ergibt sich die maximale Flussdichte im zweiten Pol zu

$$
B_{\delta 2, \max }=\frac{\Phi_{\delta 2}}{A_{\delta 2}}
$$

mit

$$
\Phi_{\delta 2}=\frac{\Phi_{\mathrm{PM} 2}-\Phi_{\sigma 1}-\Phi_{\sigma 2}}{1+\frac{R_{\delta 2}}{R_{\mathrm{PM} 2}}} .
$$

Tab. 1. Maschinendaten der Referenzmaschine

\begin{tabular}{llll}
\hline Parameter & Zeichen & Wert & Einheit \\
\hline Strangzahl & $m$ & 3 & - \\
Polpaarzahl & $p$ & 4 & - \\
Nutzahl & $N$ & 48 & - \\
Strangwindungszahl & $W$ & 12 & - \\
Maximaler Strom & $I_{\max }$ & 600 & $\mathrm{~A}$ \\
Blechpaketlänge & $l_{\mathrm{ax}}$ & 170 & $\mathrm{~mm}$ \\
Statoraußendurchmesser & $d_{\mathrm{a} 1}$ & 220 & $\mathrm{~mm}$ \\
Statorinnendurchmesser & $d_{\mathrm{i} 1}$ & 160 & $\mathrm{~mm}$ \\
Luftspalthöhe & $\delta$ & 1 & $\mathrm{~mm}$ \\
Magnetbreite & $b_{\mathrm{PM}}$ & 17,5 & $\mathrm{~mm}$ \\
Magnethöhe & $h_{\mathrm{PM}}$ & 5 & $\mathrm{~mm}$ \\
Höhe des Fügespalts & $h_{\mathrm{Spalt}}$ & 0,3 & $\mathrm{~mm}$ \\
Streustegbreite 1 & $b_{\sigma 1}$ & 1,7 & $\mathrm{~mm}$ \\
Streustegbreite 2 & $b_{\sigma 2}$ & 1,5 & $\mathrm{~mm}$ \\
Anstellwinkel 1 & $\beta_{1}$ & 33,9 & $\circ$ \\
Anstellwinkel 2 & $\beta_{2}$ & 39,8 & $\circ$ \\
Material & & & \\
Elektroblech & NO30 & & \\
Permanentmagnete & NdFeB (N35) & & \\
\hline
\end{tabular}

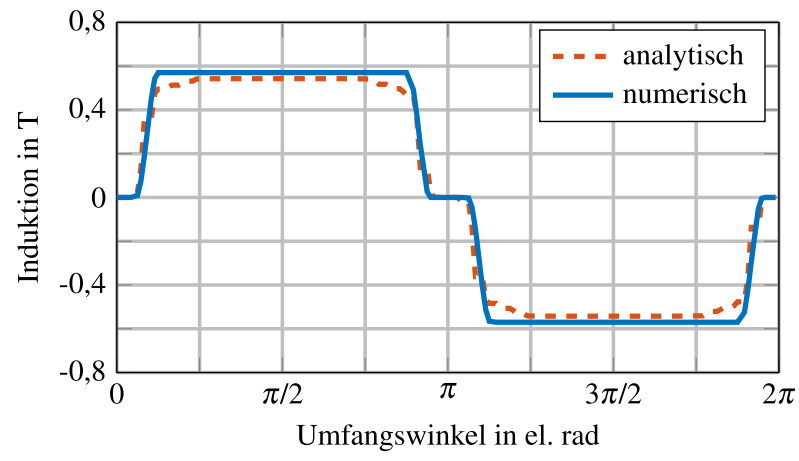

Abb. 7. Vergleich der numerisch und der analytisch ermittelten Luftspaltinduktion im Leerlauf für symmetrische Pole

Der Versatzfaktor der Induktion $\Delta B$ kann schließlich über die Differenz der maximalen Induktionen in Pol 1 und Pol 2 bezogen auf die Polbedeckungsgrade

$$
\Delta B=\frac{1}{\pi}\left[\left(\beta_{4}+\beta_{3}\right) B_{\delta 2}-\left(\beta_{2}+\beta_{1}\right) B_{\delta 1}\right]
$$

gebildet werden.

\subsection{Validierung des magnetischen Ersatznetzwerks}

Zur Validierung des vorgestellten magnetischen Ersatznetzwerks wird zunächst das Luftspaltfeld einer symmetrischen Referenzmaschine mithilfe einer magnetostatischen FEM-Berechnung im Leerlauf mit FEMAG DC berechnet. Die Maschinendaten sind in Tab. 1 zusammengefasst. Der Stator wird als magnetischer Rückschluss ausgeführt und der Vergleich des analytisch bestimmten Induktionsverlaufs mit der numerischen Lösung wird in Abb. 7 gezeigt.

Es ist zu erkennen, dass der Polbedeckungsgrad gut über die ausgelesenen Winkel identifiziert werden kann. Die maximale Amplitude des Luftspaltfelds $B_{\delta 1, \text { max }}$ wird in der analytischen Berechnung um $6 \%$ zu groß bestimmt. Begründet kann diese Differenz anhand der Vernachlässigung des magnetischen Widerstands des 


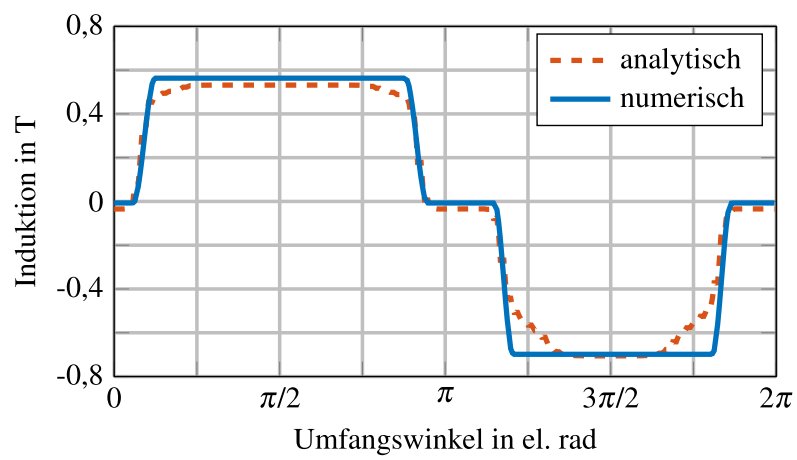

Abb. 8. Vergleich der numerisch und der analytisch ermittelten Luftspaltinduktion im Leerlauf für asymmetrische Pole

Rotorjochs. Vor allem Bereiche oberhalb der Magnete können gesättigt sein. Der magnetische Spannungsabfall in diesen Bereichen führt zu einer Reduktion der magnetischen Flussdichte im Luftspaltfeld. Nun wird eine Rotorpolweitenmodulation durchgeführt, indem die Anstellwinkel in Pol 1 aus Tab. 1 übernommen werden und in Pol zwei die Winkel $\beta_{2}=27^{\circ}$ sowie $\beta_{4}=32^{\circ}$ ausgeführt werden. Der Vergleich zwischen analytischem und numerischen Luftspaltfeldverlauf in Abb. 8 zeigt auch für die Maschine mit RPWM eine genaue Wiedergabe der Polbedeckungsgrade der Maschine. $B_{\delta 1, \max }$ wird für diese Winkelkombination um $6 \%$ höher bestimmt als in der numerischen Simulation und für $B_{\delta 2, \max }$ besteht eine Abweichung von unter $1 \%$. Über eine Fast-Fourier-Transformation (FFT) kann das Frequenzspektrum der Feldverläufe bestimmt werden. Die einzelnen Amplituden der Feldwellen werden in Abb. 9 verglichen. Es zeigt sich eine gute Übereinstimmung der Oberfeldanteile sowohl für die Maschine mit symmetrischen Polen als auch für die Maschine mit RPWM. Die Hauptfeldamplitude wird für beide Anordnungen gleichermaßen in der analytischen Berechnung überschätzt, sodass die Tendenz einer Steigerung der Hauptfeldamplitude korrekt identifiziert wird.

\subsection{Analytische Identifikation vorteilhafter \\ Winkelkombinationen}

Aus der Validierung des Verfahrens geht hervor, dass das magnetische Ersatznetzwerk hinreichend genau ist, um den Einfluss der Rotorpolweitenmodulation auf den Feldverlauf darzustellen. Somit kann dieses Verfahren genutzt werden, um eine vorteilhafte Winkelkombination auszuwählen. Ziel der RPWM ist eine Reduktion der Amplituden jener Feldanteile des Rotorfelds, die in Interaktion mit dem Statorfeld zu hohen Pendelmomenten und Anregungen von Schwingungen führen. Dies sind vor allem die Rotorfeldwellen mit der gleichen Ordnungszahl wie die Nutharmonischen nach Gleichung (10)

Zur Bewertung der Varianten wird der Gesamt-Oberwellengehalt

$$
\text { Хges }=\frac{\sqrt{\sum_{v^{\prime}} \hat{B}_{v^{\prime}}^{2}}}{\hat{B}_{p}} \text { für } v^{\prime} \neq p \text {, }
$$

der die Summe aller Oberfeldanteile bezogen auf die Hauptfeldamplitude $\hat{B}_{p}$ beschreibt, vorgestellt. Wie aus dem Vergleich der Amplitudenspektren in Abb. 9 hervorgeht, wird durch die Variation der Polweite von Pol zu Pol die Polsymmetrie gebrochen, wodurch Feldwellenanteile mit geradzahligen Ordnungszahlen entstehen. Da die Feldwellen des Strombelags in Gleichung (9) nur ungeradezahlige Ordnungen aufweisen, können auch nur Pendelmomente aus der

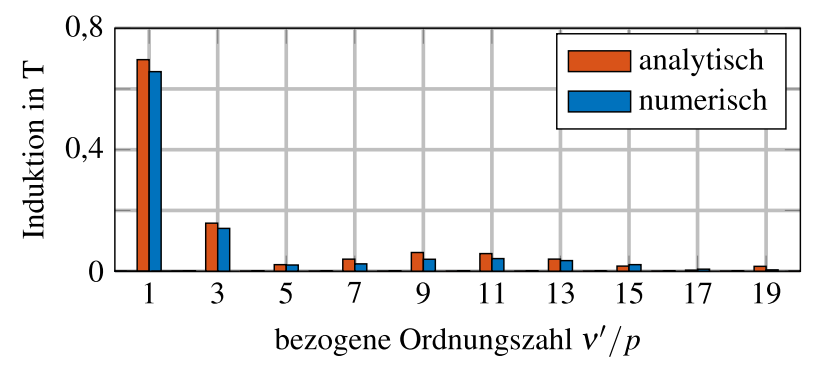

a) Symmetrische Pole

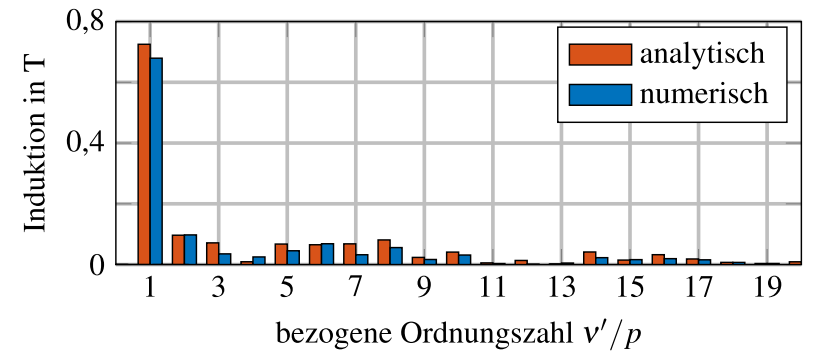

b) Asymmetrische Pole

Abb. 9. Vergleich der Amplitudenspektren des Luftspaltfeldverlaufs zwischen analytischer Rechnung und numerischer Simulation

Interaktion mit ungeradzahligen Harmonischen des Rotorfelds auftreten. Um diesen Einfluss auf die Pendelmomente abzubilden, wird der Oberwellengehalt

$$
\chi_{\text {ungerade }}=\frac{\sqrt{\sum_{v^{\prime}} \hat{B}_{v^{\prime}}^{2}}}{\hat{B}_{p}} \text { für } v^{\prime}=3 p, 5 p, 7 p, . .
$$

eingeführt. Als zusätzliche Kenngröße wird der Oberwellengehalt mit dem Wicklungsfaktor der jeweiligen Ordnungzahl bewertet gemäß

$$
\chi_{\xi}=\frac{\sqrt{\sum_{v^{\prime}} \hat{B}_{v^{\prime}}^{2} \xi_{v^{\prime}}^{2}}}{\hat{B}_{p} \xi_{p}} \text { für } v^{\prime} \neq p,
$$

um den Einfluss der Flussverkettung mit zu berücksichtigen.

Zur Identifikation einer vorteilhaften Winkelkombination wird das Rotorfeld einer Maschine nach Tab. 1 mit dem magnetischen Ersatznetzwerk berechnet, wobei gegenüber der Referenzmaschine nur die Winkelkombinationen für den zweiten Pol $\beta_{3}$ und $\beta_{4}$ variiert werden. Die identifizierten Kennfelder sind in Abb. 10 dargestellt. Zunächst ist hervorzuheben, dass eine Steigerung der Amplitude der Hauptfeldwelle durch eine RPWM möglich ist. Dieser Effekt wird umso größer, je steiler der Anstellwinkel der Magnete in Pol zwei ist. Jedoch ist für kleine Öffnungswinkel auch der Oberwellengehalt höher (vgl. Abb. 11). Aus Abb. 11 b) und c) geht hervor, dass der Oberwellengehalt der ungeradzahligen Harmonischen bzw. der verkettete Oberwellengehalt minimiert werden kann, wenn der kleinste Winkel für $\beta_{3}$ genutzt wird. Zusätzlich zum Oberwellengehalt und dem Einfluss auf die Hauptwelle wird in Abb. 10 b) die relative Abweichung auf die Rotorfeldwelle mit der Ordnung $11 p$ hervorgehoben. Dieser Feldwelle wird besondere Beachtung geschenkt, da sie im Feldspektrum der Referenzmaschine in Abb. 9 eine signifikante Amplitude aufweist und aus der Interaktion mit den 1. Nutharmonischen nach Gleichung (10) Pendelmomente erzeugen wird. Es stellt sich heraus, dass die Amplitude dieser Feldwelle durch eine RPWM 


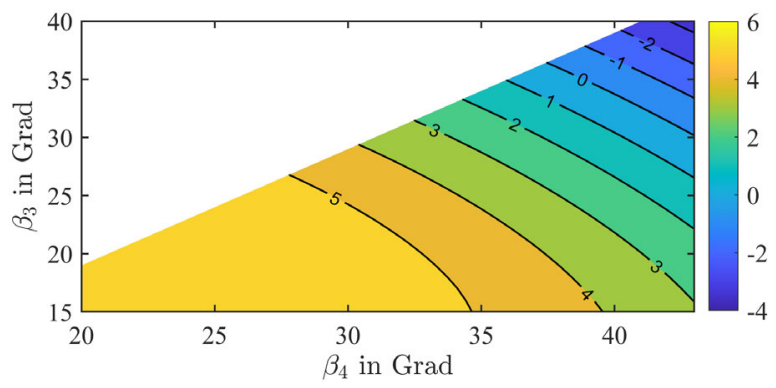

a) Relative Abweichung der Hauptfeldamplitude

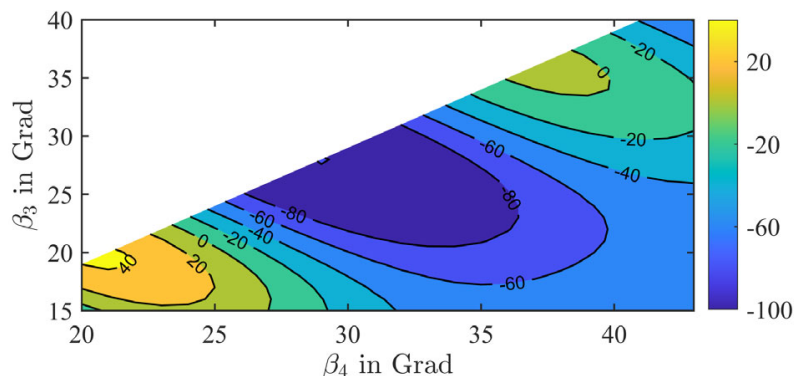

b) Relative Abweichung der Feldwelle $B_{11 \mathrm{p}}$

Abb. 10. Relative Amplitudendifferenz der Feldwellen zur symmetrischen Referenzmaschine durch Variation der Winkel $\beta_{3}$ und $\beta_{4}$ in $\%$

Tab. 2. Oberwellengehalt der Referenzmaschine sowie der Maschine mit RPWM $\left(\beta_{3}=27^{\circ}\right.$ und $\left.\beta_{4}=32^{\circ}\right)$

\begin{tabular}{llll}
\hline & $\chi_{\text {ges }}$ & $\chi_{\text {ungerade }}$ & $\chi_{\xi}$ \\
\hline Referenzmaschine & 0,277 & 0,277 & 0,210 \\
RPWM & 0,277 & 0,180 & 0,093 \\
\hline
\end{tabular}

um über $80 \%$ gegenüber der symmetrischen Referenzmaschine verringert werden kann.

Unter Berücksichtigung aller dargestellten Kennfelder und mit der Randbedingung, dass in der Praxis nur kleine Winkeldifferenzen mit $\Delta \beta=\beta_{4}-\beta_{3}=\left\{5^{\circ}-7^{\circ}\right\}$ sinnvoll umsetzbar sind, wird die Winkelkombination $\beta_{3}=27^{\circ}$ und $\beta_{4}=32^{\circ}$ als eine vorteilhafte Variante identifiziert. Diese Winkelkombination ermöglicht eine Steigerung der Hauptfeldamplitude um ca. $4 \%$, wobei die Amplituden der Feldwellen $11 p$ und $13 p$ um $90 \%$ geringer sind im Vergleich zur Maschine mit symmetrischen Polen. In Tab. 2 werden die Werte des Oberwellengehalts gegenüber gestellt. Die Maschine mit RPWM weist den gleichen Wert für den Gesamt-Oberwellengehalt $\chi_{\text {ges }}$ auf, während die Amplituden ungeradzahliger Feldanteile deutlich gegenüber der Referenzmaschine reduziert werden konnten. Dieses Ergebnis zeigt, dass durch diese Variante der RPWM ungeradzahlige Harmonische in geradzahlige Feldanteile verschoben wurden, ohne dass der Gesamt-Oberwellengehalt gestiegen ist.

\section{Einfluss der RPWM auf das Drehmomentverhalten}

Nachdem in Abschn. 3 vorteilhafte Kombinationen für eine Maschine mit variierendem Polbedeckungsgrad mithilfe des analytischen Ersatznetzwerks identifiziert worden sind, wird die ausgewählte Variante mit $\beta_{3}=27^{\circ}$ und $\beta_{4}=32^{\circ}$ mit der ungestaffelten sowie der gestaffelten Referenzmaschine anhand von FEM-Berechnungen verglichen. Die Maschinenmodelle sind in Abb. 12 dargestellt. Zunächst wird der Einfluss auf das Rastmoment im Leerlauf untersucht. An-

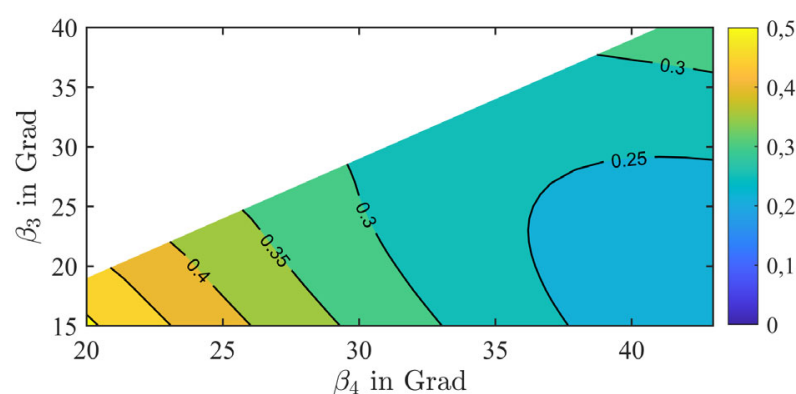

a) $\chi_{\text {ges }}$

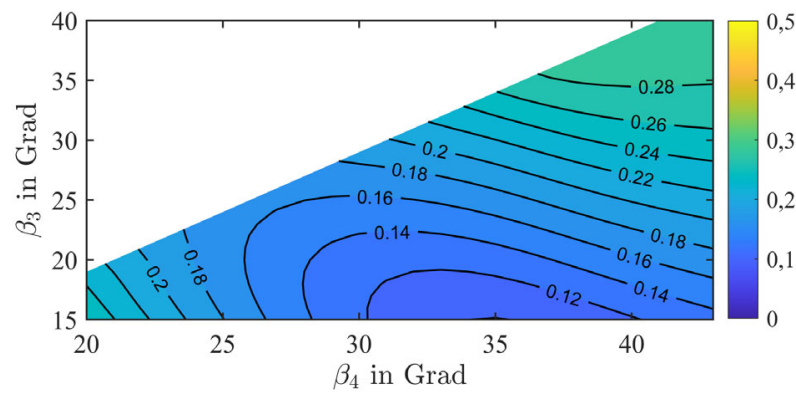

b) $\chi_{\text {ungerade }}$

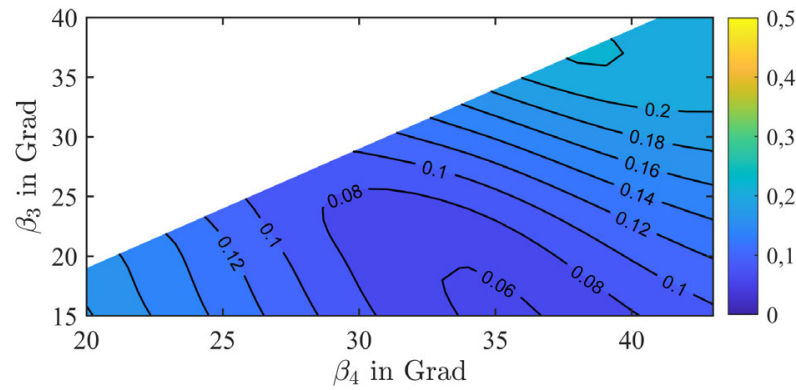

c) $\chi \xi$

Abb. 11. Einfluss einer Variation der Winkel $\beta_{3}$ und $\beta_{4}$ auf den Oberwellengehalt
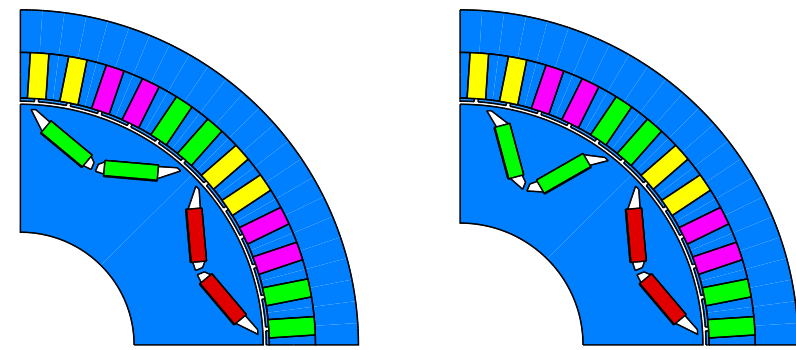

Abb. 12. FEM-Modell der Referenzmaschine (links) sowie der Machine mit RPWM (rechts)

schließend erfolgt die Bewertung des Einflusses einer RPWM im gesamten Betriebsbereich anhand eines wirkungsgradoptimierten ${ }^{1}$ Drehmoment-Drehzahl-Kennfelds.

${ }^{1}$ Wirkungsgradoptimiert bedeutet, dass im gesamten Betriebsbereich jeweils diejenige Kombination aus $i_{d}$ und $i_{q}$ gewählt wird, die die geringsten Gesamtverluste verursacht. 


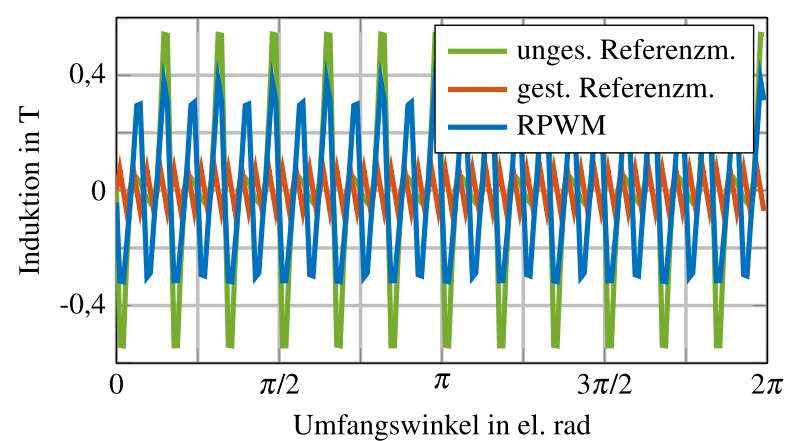

Abb. 13. Vergleich der Rastmomente im Leerlauf

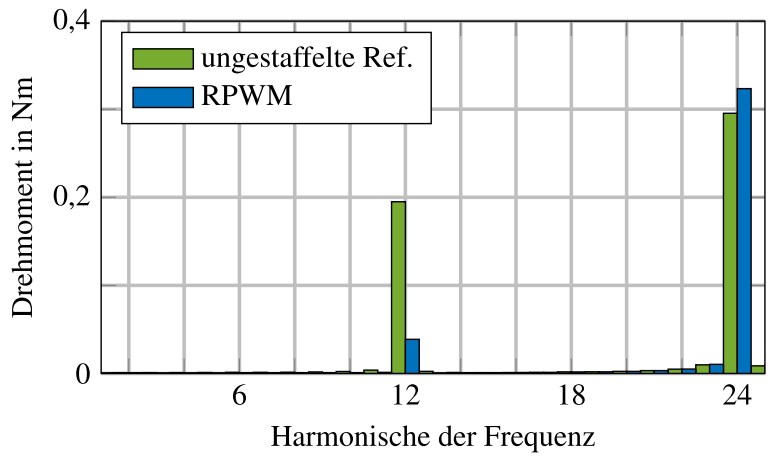

Abb. 14. Amplituden der Harmonischen des Drehmoments im Leerlauf

Die gestaffelte Maschine ist mit 4 Segmenten ausgeführt und zur Reduktion der Flussverkettung der Nutharmonischen um insgesamt eine Nutteilung gestaffelt [20]. Im Leerlauf kann somit durch die Ausführung einer Staffelung das Rastmoment von 0,95 Nm auf $0,15 \mathrm{Nm}$ reduziert werden. Dies ist in Abb. 13 dargestellt. Durch die Rotorpolweitenmodulation kann das Rastmoment gegenüber der ungestaffelten Referenzmaschine um ca. $36 \%$ auf $0,6 \mathrm{Nm}$ verringert werden. Dies wird erreicht, indem die Pendelmomente mit der Frequenz $12 f_{1}$ reduziert werden (vgl. Abb. 14). Die Pendelmomente mit der Frequenz $24 f_{1}$ werden dagegen durch die Variation der Anstellwinkel von Pol zu Pol kaum beeinflusst. Dies ist ein direktes Resultat der Beeinflussung des Rotorfelds durch asymmetrische Pole, was in Abb. 9 gezeigt wurde. So wurden die Amplituden der Feldwellen $11 p$ und $13 p$ des Rotorfelds gegenüber der ungestaffelten Referenzmaschin um 90\% verringerte.

Nun wird ein Drehmoment-Drehzahl-Kennfeld unter Einhaltung der maximalen Spannung und des maximalen Stroms berechnet. Die relative Drehmomentwelligkeit

$$
\Delta M_{\text {rel }}=\frac{M_{\text {max }}-M_{\text {min }}}{M_{\text {mittel }}}
$$

wird für jeden Arbeitspunkt berechnet und in Abb. 15 dargestellt. Beim Vergleich der Kennfelder ist festzustellen, dass die Maschine RPWM im gesamten Betriebsbereich eine um $2 \%$ bis $4 \%$ größere Drehmomomentwelligkeit als die gestaffelte Referenzmaschine aufweist. Insgesamt stellt sich die Methodik der RPWM im Bezug auf die Reduktion der Pendelmomente als wirkungsvoll heraus. Dies wird deutlich, wenn man ungestaffelte Referenzmaschine mit in den Vergleich aufnimmt. Dazu werden die Drehmomente und die relative Drehmomentwelligkeit für die Arbeitspunkte AP1 und AP2 an der Grenzkennlinie in Tab. 3 zusammengefasst.

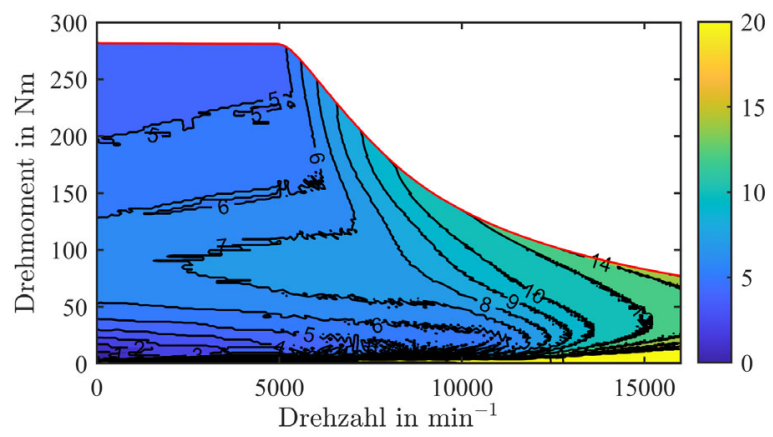

a) Gestaffelte Referenzmaschine

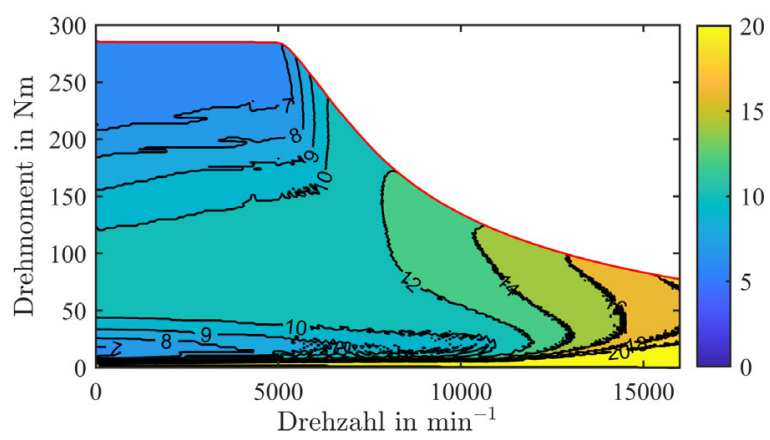

b) Maschine RPWM

Abb. 15. Relative Drehmomentwelligkeit $\Delta \boldsymbol{M}_{\text {rel }}$ in \% im motorischen Drehmoment-Drehzahl-Kennfeld

Tab. 3. Vergleich des Drehmomentverhaltens in unterschiedlichen Arbeitspunkten an der Grenzkennlinie; in den Klammern ist die relative Drehmomentwelligkeit angegeben

\begin{tabular}{llll}
\hline Maschine & Parameter & AP1 & AP2 \\
\hline & $n$ & $5000 \mathrm{~min}^{-1}$ & $16000 \mathrm{~min}^{-1}$ \\
\hline Ref. gestaffelt & $i_{\mathrm{d}}$ & $-581 \mathrm{~A}$ & $-538 \mathrm{~A}$ \\
& $i_{\mathrm{q}}$ & $613 \mathrm{~A}$ & $140 \mathrm{~A}$ \\
& $M$ & $281 \mathrm{Nm}(5 \%)$ & $77 \mathrm{Nm}(16 \%)$ \\
Ref. ungestaffelt & $i_{\mathrm{d}}$ & $-604 \mathrm{~A}$ & $-553 \mathrm{~A}$ \\
& $i_{\mathrm{q}}$ & $589 \mathrm{~A}$ & $140 \mathrm{~A}$ \\
& $M$ & $288 \mathrm{Nm}(21 \%)$ & $80 \mathrm{Nm}(60 \%)$ \\
RPWM & $i_{\mathrm{d}}$ & $-610 \mathrm{~A}$ & $-583 \mathrm{~A}$ \\
& $i_{\mathrm{q}}$ & $540 \mathrm{~A}$ & $135 \mathrm{~A}$ \\
& $M$ & $285 \mathrm{Nm}(7 \%)$ & $78 \mathrm{Nm}(19 \%)$ \\
\hline
\end{tabular}

Der Arbeitspunkt AP1 stellt den Eckpunkt der Maschine dar, an dem die Maschine vom Konstantfluss- in dem Feldschwächbereich übergeht. Hier kann zum einen die Drehmomentwelligkeit von $21 \%$ für die ungestaffelte Referenzmaschine auf 7\% durch Ausführung einer RPWM reduziert werden. Dies ist wiederum auf die Verringerung der Pendelmomente mit $12 f_{1}$ zurückzuführen, wie in Abb. 16 dargestellt ist. Zum anderen kann im Gegensatz zur gestaffelten Variante ein um 1,5\% höheres maximales Drehmoment bei gleichem Magnetvolumen erreicht werden. Somit kann hier ein Einsparpotenzial für das Magnetmaterial verzeichnet werden, wenn das gleiche maximale Drehmoment wie für die gestaffelte Referenzmaschine erzielt werden soll.

Im Arbeitspunkt AP2 beträgt die relative Drehmomentwelligkeit für die ungestaffelte Variante 60\%. Mithilfe der RPWM kann 


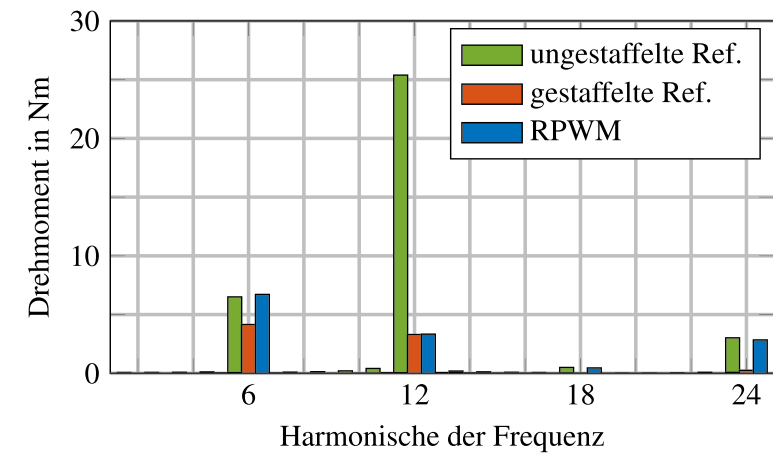

Abb. 16. Amplituden der Harmonischen des Drehmoments im AP1

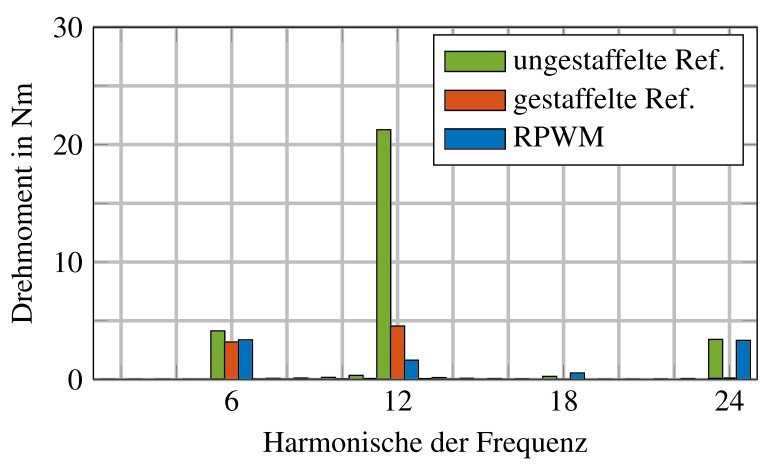

Abb. 17. Amplituden der Harmonischen des Drehmoments im AP2

Kraftberechnung Strukturdynamik Akustisches Modell

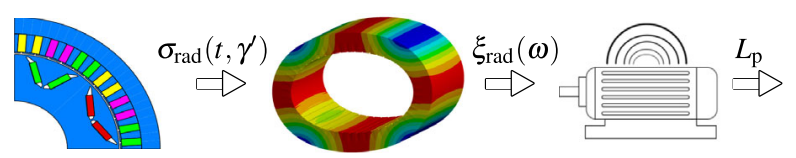

Abb. 18. Methodik zur Berechnung der Geräuschemissionen

$\Delta M_{\text {rel }}=19 \%$ erreicht werden. Die Modifikation führt zur Reduktion von $\hat{M}_{12 f 1}=21,3 \mathrm{Nm}$ auf $1,6 \mathrm{Nm}$ (vgl. Abb. 17). Während die Amplitude der Pendelmomente mit $12 f_{1}$ für die gestaffelte Maschine um Faktor den 3 größer ist, führt die Staffelung zusätzlich zur Auslöschung der Pendelmomente mit $24 f_{1}$, wodurch die resultierende Drehmomentwelligkeit um 3\% geringer ist als bei der Maschine RPWM.

\section{Berechnung der abgestrahlten Geräusche}

Nachdem in Abschn. 4 gezeigt werden konnte, dass mit einer Maschine mit Rotorpolweitenmodulation ein nahezu äquivalentes Drehmomentverhalten wie für eine gestaffelte Maschine erreicht werden kann, wird nun der Einfluss der Rotormodifikation auf die Geräuschemission dargestellt. Zur Bestimmung der abgestrahlten Geräusche wird ein analytisch-numerisch-gekoppelter Ansatz gewählt, der eine zeiteffiziente Berechnung ermöglicht [23, 24]. Der Berechnungsablauf ist in Abb. 18 dargestellt.

Zunächst werden mithilfe einer elektromagnetischen 2D-FEMBerechnung die Kräfte identifiziert, die auf die Statorzahnköpfe wirken. Die entstehenden zeit- und ortsabhängigen Kraftdichtewellen $\sigma_{\text {rad }}$ führen zur Verformung des mechanischen Modells, sodass über eine modale Superposition die radialen Schwingungen an der Oberfläche des Stators $\xi(\omega)$ bestimmt werden können. Mithilfe ei-
Tab. 4. Materialeigenschaften des mechanischen Modells

\begin{tabular}{lll}
\hline Komponente & Dichte in $\mathrm{kg} / \mathrm{m}^{3}$ & E-Modul in Pa \\
\hline Statorblech & 7600 & $1,8 \cdot 10^{11}$ \\
Wicklung & 7467 & $3,0 \cdot 10^{9}$ \\
\hline
\end{tabular}
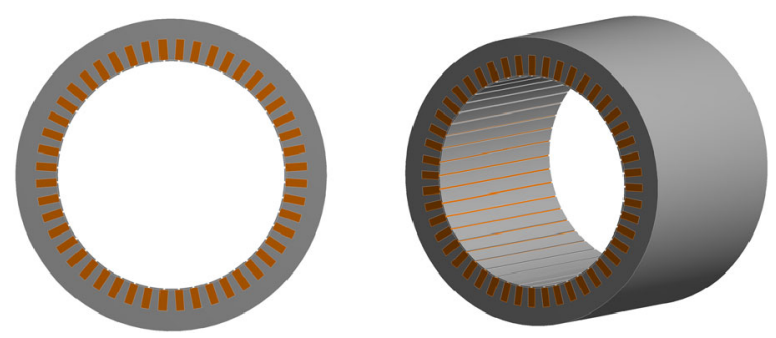

Abb. 19. 3D-FEM-Modell für die mechanische Berechnung

Tab. 5. Eigenfrequenzen der ersten vier radialen Moden

\begin{tabular}{lllll}
\hline$r$ & 0 & 2 & 3 & 4 \\
\hline$f_{\text {eigen, } r \text { in Hz }}$ & 5538 & 736 & 1985 & 3625 \\
\hline
\end{tabular}

nes akustischen Modells, welches das Abstrahlverhalten beschreibt, kann der abgestrahlte Schalldruckpegel $L_{p}$ bestimmt werden. Das hier anwendete akustische Modell ist ein endlicher Zylinderstrahler [24]. Mit den Materialdaten in Tab. 4 wird ein strukturmechanisches 3D-Modell in Ansys Mechanical aufgebaut, welches in Abb. 19 dargestellt ist. Die Materialdaten der Wicklung entsprechen einem homogenisierten Material aus Kupfer und Isolation.

Das mechanische Modell des Stators ist für alle dargestellten Maschinen gleich, da nur eine Modifikation am Rotor ausgeführt wird. Ein Gehäuse sowie eine Lagerung wird für diesen Vergleich nicht hinzugefügt, sodass Torsionsmoden in der Geräuschberechnung hier nicht berücksichtigt werden. Mit dem 3D-FEM-Modell wird lediglich die Modalwertmatrix des Stators berechnet. Die Schwingungsamplituden an der Außenkontur werden anschließend, wie in [24] beschrieben, mittels modaler Superposition berechnet. Die Eigenfrequenzen der ersten vier radialen Moden sind in Tab. 5 zusammengefasst.

Zur Bewertung des akustischen Verhaltens der drehzahlvariablen Maschinen wird ein Hochlauf berechnet. So ist es möglich zu bestimmen, bei welcher Drehzahl und mit welcher Frequenz Resonanzerscheinungen auftreten. Der Hochlauf wird mit einer konstanten Bestromung von $i_{\mathrm{d}}=\sqrt{2} \cdot-100 \mathrm{~A}$ und $i_{\mathrm{q}}=\sqrt{2} \cdot 100 \mathrm{~A}$ simuliert, wobei sichergestellt wird, dass die Spannungsgrenze im gewählten Drehzahlbereich eingehalten wird. Verglichen wird der abgestrahlte Gesamtschalldruckpegel an der Oberfläche der Maschine, welcher über die logarithmische Addition der Schalldruckpegel $L_{p, k}$ aller $k$ Schwingungen

$$
L_{p, \text { ges }}=10 \log _{10}\left(\sum_{k} 10^{\frac{L_{p, k}}{10}}\right)
$$

definiert ist [24]. Der Verlauf von $L_{p \text {,ges }}$ über der Drehzahl ist in Abb. 20 dargestellt. Zusätzlich sind die Ordnungen der radialen und der axialen Schwingungsmoden $(r, q)$ eingetragen, die resonanznah durch die magnetischen Kräfte angeregt werden, was zu lokalen Überhöhungen des Schalldruckpegels führt. 


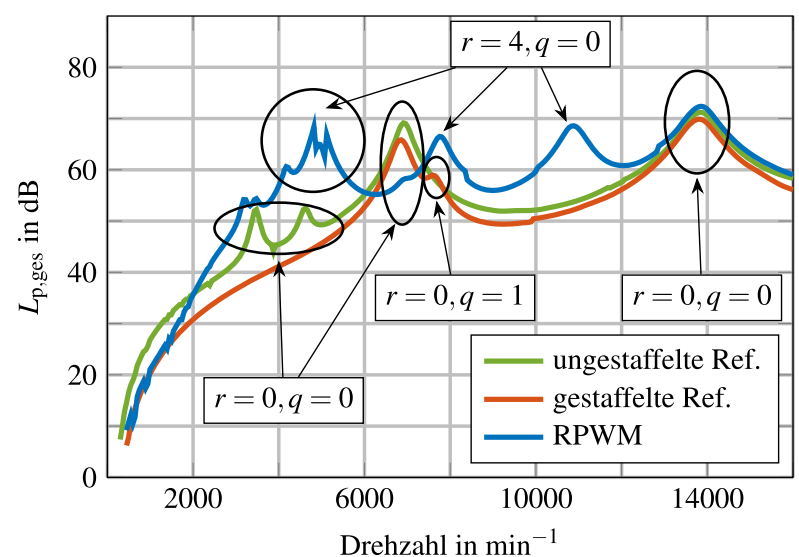

Abb. 20. Gesamtschalldruckpegel im Hochlauf mit konstanter Bestromung

Beim Vergleich der gestaffelten Referenzmaschine mit der ungestaffelten Referenzmaschine fällt zunächst auf, dass die Staffelung zu einer Reduzierung des Gesamtschalldruckpegels im kompletten Drehzahlbereich führt. So treten bei Staffelung im niedrigen Drehzahlbereich zwischen $3000 \mathrm{~min}^{-1}$ und $6000 \mathrm{~min}^{-1}$ keine Resonanzen auf. Die Anregungen der Eigenfrequenzen der Atemmode $(r=0, q=0)$ bei $6900 \mathrm{~min}^{-1}$ und bei $13900 \mathrm{~min}^{-1}$ sind weiterhin für die gestaffelte Maschine sowie für die ungestaffelte Maschine zu erkennen. Hier wird jeweils eine Reduktion des Schalldruckpegels mithilfe der Staffelung erzielt. Zusätzlich wird bei Staffelung bei $7600 \mathrm{~min}^{-1}$ die Mode $r=0, q=1$ angeregt, was aus der ungleichmäßigen Krafteinprägung über die axiale Länge aufgrund der Staffelung des Rotors resultiert.

Wird nun der Hochlauf für die Maschine RPWM berechnet, ist zu beobachten, dass die Resonanz bei $13900 \mathrm{~min}^{-1}$ zwar einen höheren Schalldruckpegel aufweist, jedoch die Überhöhung des Schalldruckpegels bei $6900 \mathrm{~min}^{-1}$ deutlich durch die RPWM reduziert wird. So kann für diese Drehzahl eine Reduktion von $8 \mathrm{~dB}$ gegenüber der gestaffelten und von $12 \mathrm{~dB}$ gegenüber der ungestaffelten Maschine erzielt werden. Allerdings sind zusätzliche Anregungen der Eigenfrequenz der Mode $r=4, q=0$ zu verzeichnen, sodass zusätzliche lokale Maxima und insgesamt ein höherer abgestrahlter Schalldruckpegel berechnet wird.

Zur genaueren Bewertung des Ursprungs der Resonanzen werden die Campbell-Diagramme in Abb. 21 herangezogen. Aufgrund der Frequenzabhängigkeit der Kraftwellen sind diese als lineare Strahlen abgebildet. Dadurch kann bei einer Resonanzerscheinung auf die verantwortliche Kraftanregung zurück geschlossen werden. So kann für die gestaffelte Maschine bei der Drehzahl $6900 \mathrm{~min}^{-1}$ die Hauptkraftamplitude $r=0, f_{r}=12 p \cdot n$ zur Anregung der Eigenfrequenz $f_{\text {eigen, } 0}=5530 \mathrm{~Hz}$ identifiziert werden. Bei $13900 \mathrm{~min}^{-1}$ ist die Kraftanregung mit der Ordnung $r=0, f_{r}=6 p \cdot n$ für die Überhöhung des Schalldruckpegels verantwortlich. Tangentiale Kraftanregungen mit derselben Ordnung und Frequenz sind auch für die Pendelmomente identifiziert worden (vgl. Abb. 16).

Im Campbell-Diagramm der Maschine RPWM ist hervorzuheben, dass sich zum einen die Anzahl der Kraftwellen gegenüber Abb. 21a) verdoppelt hat. Dies ist auf die Entstehung von zusätzlichen geradzahligen Harmonischen im Luftspaltfeld zurückzuführen (vgl. Abb. 9). Zum anderen bewirkt das Vorhandensein der geradzahligen Harmonischen die Möglichkeit der Ausprägung einer Kraftform mit $r=4$. Die Anregung der Eigenfrequenz dieser Mode mit $f_{\text {eigen, } 4}=3625 \mathrm{~Hz}$ dominiert die Geräuschemission dieser Maschine. So kann beispielsweise die Ursache für die Resonanz bei $4800 \mathrm{~min}^{-1}$

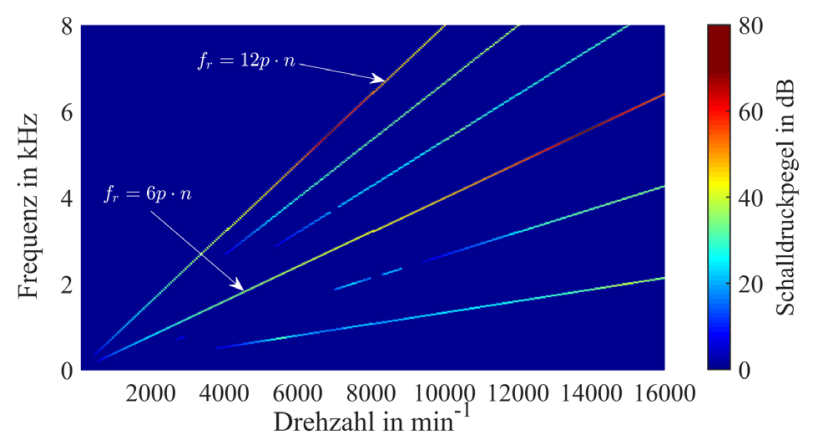

a) Gestaffelte Referenzmaschine

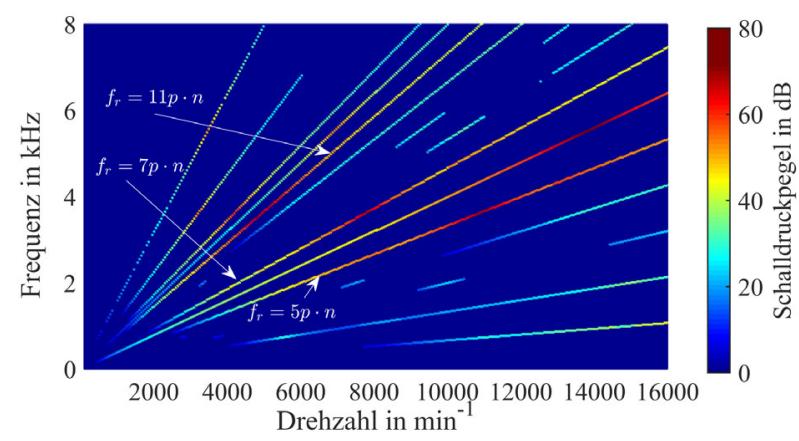

b) Maschine mit RPWM

\section{Abb. 21. Schalldruckpegel im Campbell-Diagramm}

in der Kraftwelle $r=4, f_{\mathrm{r}}=11 p \cdot n$ gefunden werden und bei $10900 \mathrm{~min}^{-1}$ bewirkt die Kraftanregung $r=4, f_{\mathrm{r}}=5 p \cdot n$ eine lokale Überhöhung des Schalldruckpegels. Die Anregung der Atemmode ist dagegen bei der Maschine mit RPWM in einem großen Drehzahlbereich nicht entscheidend für den resultierenden Gesamtschalldruckpegel.

\section{Zusammenfassung}

In diesem Artikel ist der Einfluss einer Rotorpolweitenmodulation auf das Betriebsverhalten einer permanentmagneterregten Synchronmaschine untersucht worden. Dazu ist ein magnetisches Ersatznetzwerk vorgestellt worden, mit dem vorteilhafte Winkelkombinationen identifiziert werden können. Anschließend wurde eine Maschine mit RPWM ausgewählt und mit einer gestaffelten Referenzmaschine verglichen. Die Haupterkenntnisse sind im Folgenden zusammengefasst:

- Bei der Ausführung einer Rotorpolweitenmodulation wird die Polsymmetrie gebrochen, wodurch das Luftspaltfeld des Rotors zusätzlich geradzahlige Harmonische aufweist.

- Außerdem ist es möglich, durch eine der Variation des Anstellwinkels von Pol zu Pol die Hauptfeldamplitude gegenüber einer symmetrischen Anordnung zu steigern.

- Dadurch kann eine Maschine mit Rotorpolweitenmodulation ein höheres maximales Drehmoment aufweisen als die gestaffelte Referenzmaschine bei ähnlich geringer Drehmomentwelligkeit. Somit kann bei der Dimensionierung einer Maschine mit dem gleichen Maximalmoment zusätzlich zum Fertigungsschritt der Staffelung auch Magnetmaterial eingespart werden.

- Da geradzahlige Feldwellen keinen Einfluss auf die Drehmomentbildung besitzen, eignet sich die RPWM zur Reduktion der Pendelmomente im gesamten Betriebsbereich einer Traktionsmaschine. Mit einer zusätzlichen Modifikation der Stator- oder Rotorgeometrie könnte so die Drehmomentwelligkeit weiter verringert werden, sodass das Ausführen einer Staffelung nicht mehr nötig ist. 
- Aufgrund der geradzahligen Harmonischen des Luftspaltfelds kann eine Maschine mit RPWM zusätzliche radiale Kraftanregungen ausprägen. Dies führt zur Verdoppelung der Anzahl der potenziellen radialen Kraftwellen sowie zu einer zusätzlichen Anregung der Mode $r=p$. Der Einfluss dieser Mode dominiert bei der untersuchten Beispielmaschine den abgestrahlten Schalldruckpegel und führt zu insgesamt höheren Geräuschemissionen im Vergleich zu der gestaffelten Maschine.

- Daher sollte bei der Dimensionierung einer Maschine mit RPWM berücksichtigt werden, dass die Amplituden der geradzahligen Harmonischen des Luftspaltfelds begrenzt werden müssen.

Funding Note Open Access funding enabled and organized by Projekt DEAL.

Hinweis des Verlags Der Verlag bleibt in Hinblick auf geografische Zuordnungen und Gebietsbezeichnungen in veröffentlichten Karten und Institutsadressen neutral.

Open Access Dieser Artikel wird unter der Creative Commons Namensnennung 4.0 International Lizenz veröffentlicht, welche die Nutzung, Vervielfältigung, Bearbeitung, Verbreitung und Wiedergabe in jeglichem Medium und Format erlaubt, sofern Sie den/die ursprünglichen Autor(en) und die Quelle ordnungsgemäß nennen, einen Link zur Creative Commons Lizenz beifügen und angeben, ob Änderungen vorgenommen wurden. Die in diesem Artikel enthaltenen Bilder und sonstiges Drittmaterial unterliegen ebenfalls der genannten Creative Commons Lizenz, sofern sich aus der Abbildungslegende nichts anderes ergibt. Sofern das betreffende Material nicht unter der genannten Creative Commons Lizenz steht und die betreffende Handlung nicht nach gesetzlichen Vorschriften erlaubt ist, ist für die oben aufgeführten Weiterverwendungen des Materials die Einwilligung des jeweiligen Rechteinhabers einzuholen. Weitere Details zur Lizenz entnehmen Sie bitte der Lizenzinformation auf http://creativecommons.org/licenses/by/4.0/deed.de.

\section{Literatur}

1. Putri, A. K., Rick, S., Franck, D., Hameyer, K. (2016): Application of sinusoidal field pole in a permanent-magnet synchronous machine to improve the NVH behavior considering the MTPA and MTPV operation area. IEEE Trans. Ind. Appl., 52(3), 2280-2288.

2. Cassat, A., et al. (2012): A practical solution to mitigate vibrations in industrial PM motors having concentric windings. IEEE Trans. Ind. Appl., 48(5), 1526-1538.

3. Gan, C., Wu, J., Shen, M., Yang, S., Hu, Y., Cao, W. (2015): Investigation of skewing effects on the vibration reduction of three-phase switched reluctance motors. IEEE Trans. Magn., 51(9), 1-9.

4. England, M., Ponick, B. (2019): Automatisierter Entwurf von Haarnadelwicklungen anhand tabellarischer Belegungspläne. E\&l, Elektrotech. Inf.tech., 136(2), 159-167.

5. Blum, J., Merwerth, J., Herzog, H. (2014): Investigation of the segment order in step-skewed synchronous machines on noise and vibration. In 2014 4th international electric drives production conference (EDPC), Nürnberg, Deutschland (S. 303-308).

6. Wang, S., Hong, J., Sun, Y., Cao, H. (2020): Effect comparison of zigzag skew PM pole and straight skew slot for vibration mitigation of PM brush DC motors. IEEE Trans. Ind. Electron., 67(6), 4752-4761.

7. Sim, J., Jung, J., Kim, Y., Lee, B., Hong, J. (2012): Optimum design of SPMSM with concentrated windings and unequal tooth widths for EPS. In 2012 IEEE vehicle powe and propulsion conference (VPPC), Seoul, Südkorea (S. 191-195)
8. Herlina, H., Setiabudy, R., Rahardjo, A. (2017): Influence of permanent magnet and width of stator slot to cogging torque reduction in PMSG using anti-notch and cutting edge method. In 2017 15th international conference on quality in research (QiR): international symposium on electrical and computer engineering, Bali, Indonesien ( $S$. 408-413).

9. Ren, W. (2017): A technique of torque ripple compensation for interior permanent magnet machines by using an asymmetrical V-type rotor configuration. In IECON 2017 - 43rd annual conference of the IEEE industrial electronics society Beijing, China (S. 8744-8749).

10. Sayed, E., Yang, Y., Bilgin, B., Bakr, M. H., Emadi, A. (2019): A comprehensive review of flux barriers in interior permanent magnet synchronous machines. IEEE Access, 7, 149168-149181.

11. Zhou, T., Shen, J. (2017): Cogging torque and operation torque ripple reduction of interior permanent magnet synchronous machines by using asymmetric flux-barriers. In 2017 20th international conference on electrical machines and systems (ICEMS), Sydney, Australien (S. 1-6).

12. Kim, K. (2014): A novel method for minimization of cogging torque and torque ripple for interior permanent magnet synchronous motor. IEEE Trans. Magn., 50(2), 793-796.

13. Seo, U., Chun, Y., Choi, J., Han, P., Koo, D., Lee, J. (2011): A technique of torque ripple reduction in interior permanent magnet synchronous motor. IEEE Trans. Magn., 47(10), 3240-3243.

14. Du, Z. S., Lipo, T. A. (2020): Reducing torque ripple using axial pole shaping in interior permanent magnet machines. IEEE Trans. Ind. Appl., 56(1), 148-157.

15. Liang, J., Parsapour, A., Moallem, M., Fahimi, B. (2019): Asymmetric rotor surface design in interior permanent magnet synchronous motors for torque ripple mitigation. In 2019 IEEE international electric machines and drives conference (IEMDC), San Diego, USA (S. 727-732).

16. Bianchi, N., Bolognani, S., Bon, D., Dai Pre, M. (2009): Rotor flux-barrier design for torque ripple reduction in synchronous reluctance and PM-assisted synchronous reluctance motors. IEEE Trans. Ind. Appl., 45(3), 921-928.

17. Chen, Q., Xu, G., Liu, G., Zhao, W., Liu, L., Lin, Z. (2018): Torque ripple reduction in five-phase IPM motors by lowering interactional MMF. IEEE Trans. Ind. Electron., 65(11), 8520-8531.

18. Andresen, J., Vip, S., Mertens, A., Paulus, S. (2020): Compensation of the radial and circumferential mode 0 vibration of a permanent magnet electric machine based on an experimental characterisation. In 2020 22nd European conference on power electronics and applications (EPE'20 ECCE Europe), Lyon, Frankreich (S. 1-9).

19. Farshadnia, M., Pouramin, A., Dutta, R., Fletcher, J. E. (2017): Airgap magnetic field estimation for IPM rotors considering their non-uniform local saturation. In 2017 20th international conference on electrical machines and systems (ICEMS), Sydney, Australien (S. 1-6).

20. Müller, G., Vogt, K., Ponick, B. (2008): Berechnung elektrischer Maschinen. Weinheim: Wiley-VCH.

21. Müller, G., Ponick, B. (2009): Theorie elektrischer Maschinen. Weinheim: Wiley-VCH.

22. Hofmann, A., Qi, F., Lange, T., De Doncker, R. W. (2014): The breathing mode-shape 0 : is it the main acoustic issue in the PMSMs of today's electric vehicles. In 2014 17 th international conference on electrical machines and systems (ICEMS), Hangzhou, China (S. 3067-3073)

23. Vip, S., Hollmann, J., Ponick, B. (2019): NVH-simulation of salient-pole synchronous machines for traction applications. In 2019 international Aegean conference on electrical machines and power electronics (ACEMP) \& 2019 international conference on optimization of electrical and electronic equipment (OPTIM), Istanbul, Türkei (S. 246-253).

24. Braunisch, D., Ponick, B., Bramerdorfer, G. (2013): Combined analytical-numerical noise calculation of electrical machines considering nonsinusoidal mode shapes. IEEE Trans. Magn., 49(4), 1407-1415. 


\section{Autoren}

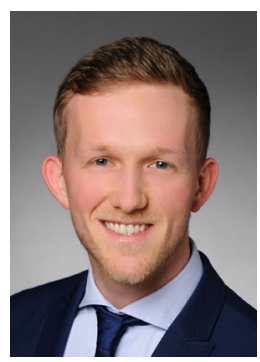

\section{Marc England}

studierte Energietechnik an der Leibniz Universität Hannover, Deutschland und erhielt im Januar 2018 den Master of Science. Im Anschluss an sein Studium ist er als wissenschaftlicher Mitarbeiter am Institut für Antriebsysteme und Leistungselektronik der Leibniz Universität Hannover angestellt worden. Seine Forschungsschwerpunkte liegen im Bereich Dimensionierung von Haarnadelwicklungen und Reduzierung der Drehmomentwelligkeit sowie Untersuchung des akustischen Verhaltens von Traktionsmaschinen.

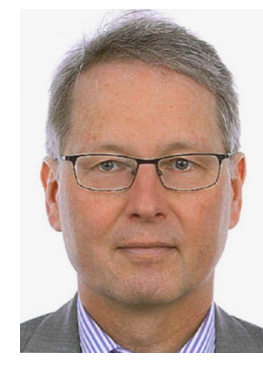

\section{Bernd Ponick}

schloss sein Studium der Elektrotechnik an der Universität Hannover, Deutschland, ab. Anschließend war er als wissenschaftlicher Mitarbeiter am Institut für Elektrische Maschinen und Antriebe tätig und wurde dort 1994 promoviert. In den folgenden Jahren arbeitete er als Entwicklungsingenieur, Berechnungsleiter und schließlich als Technischer Leiter im Berliner Dynamowerk der Siemens AG. 2003 folgte er einem Ruf auf den Lehrstuhl für Antriebssysteme der Leibniz Universität Hannover und ist seitdem einer der beiden Leiter des dortigen Instituts für Antriebssysteme und Leistungselektronik. Von 2007 bis 2016 war er als Chairman von IEC TC2 für die internationale Normung auf dem Gebiet der rotierenden elektrischen Maschinen verantwortlich. 